\title{
Curvature of the total electron density at critical coupling
}

\author{
A. Galindo, ${ }^{1,2}$ I. Nagy, ${ }^{2,3}$ R. Díez Muiño, ${ }^{2,4}$ and P. M. Echenique ${ }^{2,4,5}$ \\ ${ }^{1}$ Departamento de Física Teórica, Facultad de Física, Universidad Complutense, 28040 Madrid, Spain \\ ${ }^{2}$ Donostia International Physics Center DIPC, P. Manuel de Lardizabal 4, 20018 San Sebastián, Spain \\ ${ }^{3}$ Department of Theoretical Physics, Institute of Physics, Technical University of Budapest, H-1521 Budapest, Hungary \\ ${ }^{4}$ Unidad de Física de Materiales, Centro Mixto CSIC-UPV/EHU, P. Manuel de Lardizabal 3, 20018 San Sebastián, Spain \\ ${ }^{5}$ Departamento de Física de Materiales, Facultad de Químicas UPV/EHU, Apartado 1072, 20080 San Sebastián, Spain
}

(Received 14 March 2005; revised manuscript received 28 July 2005; published 15 September 2005)

\begin{abstract}
A structural property of the spherically averaged total charge density $\rho_{\lambda}(r)$ of a degenerate electron gas distorted by the action of an attractive, screened potential $\lambda V(r)$ is the subject of this study. The total charge density is described as a sum over occupied bound and scattering wave functions of independent electrons moving in the effective central field. It is shown that the second radial derivative $\rho_{\lambda}^{\prime \prime}(0)$ is a differentiable function of $\lambda$ for any $\lambda \geqslant 0$. The smooth behavior implies that this curvature $\rho_{\lambda}^{\prime \prime}(0)$ does not provide a criterion for the bound-to-free transition in metallic environments.
\end{abstract}

DOI: $10.1103 /$ PhysRevB.72.125113

PACS number(s): 71.10.Ca, 71.55.-i

Screening is a fundamental property of an electron gas, the prototype many-electron model of metals. When embedding an external charge into it, the electrons redistribute themselves so as to completely shield the charge at large distances. The most accurate theoretical method currently applied to this fundamental problem is based on density func-

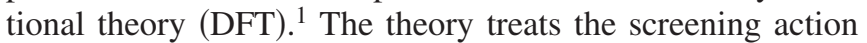
by using a grand-canonical ensemble for the electron gas with constant chemical potential and a variable number of constituents.

In the Kohn-Sham (KS) scheme, ${ }^{2}$ the physical groundstate density $\rho(r)$ is constructed from one-particle wave functions by summing over occupied states. ${ }^{3}$ For a given density of the screening environment $\rho_{0}$, and depending on the magnitude of the attractive external charge, the total density consists of bound $(b d)$ and scattering ( $s c)$ states. ${ }^{4,5}$ Detailed knowledge of these wave functions is useful in the analysis of various problems related to the embedding of moving ions in a metallic environment. ${ }^{6-8}$

Theoretical information on the structural properties of the density $\rho(r)$ other than its well-known behavior,

$$
\rho^{\prime}(0)=-2 Z \rho(0),
$$

at a nucleus with charge $Z,{ }^{9-11}$ is important in various fields of physics and chemistry. The above cusp condition does not depend on single-particle energies and also holds, separately, for bound and scattering states.

Peierls ${ }^{12}$ investigated the possibility of discontinuity in the physical variable $\rho(0)=\rho_{\mathrm{bd}}(0)+\rho_{\mathrm{sc}}(0)$ when a new bound state is created. He found a smooth behavior of $\rho(0)$ at critical coupling. The smooth behavior was also demonstrated recently by an explicit calculation based on the Hulthén potential. ${ }^{13}$ Notice that it is the determinant form of the many-body wave function, imposed by the Pauli exclusion principle, the one that yields the smooth behavior of the averaged ground-state quantity. ${ }^{12}$ The analytical character of the density matrix in the many-body context ${ }^{14}$ has been also analyzed as a function of the coupling constant $\lambda$ of a short- range potential $\lambda V(r)$, by considering only $s$-type $(l=0)$ states.

For the particular case of a closed-shell atom without the presence of a screening environment, a closed expression for the second derivative of the electron density was deduced in Ref. 15 from the radial solutions of $s$ and $p(l=1)$ occupied bound orbitals in the KS scheme.

In a bare Coulomb potential, and after making the angular average of the probability density of scattering states $g_{\mathrm{sc}}(r)$, the curvature at the origin has the following simple form: ${ }^{16}$

$$
g_{\mathrm{sc}}^{\prime \prime}(0)=4 Z^{2} g_{\mathrm{sc}}(0) .
$$

This is, quite remarkably, independent of the scattering energy. Again, both $s$ and $p$ contributions were added up to obtain this result. In the same potential, if the probability density of bound states $g_{\text {bd }}(r)$ is described only with $s$-type states, one gets an energy-dependent expression

$$
g_{\text {bd }}^{\prime \prime}(0)=\frac{2}{3} Z^{2}\left(5+\frac{1}{n^{2}}\right) g_{\text {bd }}(0),
$$

where $n$ is the principal quantum number.

Both the $g_{\text {sc }}(0)$ and $g_{\text {bd }}(0)$ probability densities satisfy Eq. (1). At vanishing one-particle binding energy $(n \rightarrow \infty)$, the prefactor of $g_{\text {bd }}(0)$ is $(10 / 3) Z^{2}$. The difference between $4 Z^{2}$ [Eq. (2)] and (10/3) $Z^{2}$ [Eq. (3)] seems to indicate a discontinuous change in the curvature of the electronic density when approaching the zero-energy state from either the bound or the scattering side. This could, according to Ref. 16, provide a criterion for the bound-to-free transition in charged systems. Stimulated by this appealing possibility, we have focused on the study of the curvature in a different context, that of electrons moving in a central screened potential.

To mark the difference, we shall distinguish in the following the electronic density obtained by summing over the occupied electronic states of a real screening problem $\left(\rho_{\mathrm{bd}}\right.$ and $\rho_{\mathrm{sc}}$ ) from the probability densities $\left(g_{\mathrm{bd}}\right.$ and $g_{\mathrm{sc}}$ ) obtained by using states of a given central field. The derivatives of $g(r)$, 
a single-particle probability, may have relevance when dealing with the screening problem in metal-insulator transitions where the abrupt disappearance of the metallic character gives rise to the appearance of an atomic limit with a fixed number of electrons in the unit cell. ${ }^{17}$

The main goal of this work is thus a rigorous analysis of $\rho^{\prime \prime}(0)$ and, more specifically, of its behavior as a function of the coupling constant $\lambda$. We will show that the angularaveraged charge density $\rho_{\lambda}(r)$ of a degenerate paramagnetic electron gas distorted by the action of a local short-range potential $\lambda V(r)$, as well as the two radial derivatives $\rho_{\lambda}^{\prime}(0)$ and $\rho_{\lambda}^{\prime \prime}(0)$, are differentiable functions of $\lambda$ for any $\lambda \geqslant 0$. There are no discontinuities in $\lambda$ that could be used as imprints for the appearance of bound states.

In the following, we simply sketch the lines along which one can rigorously prove such differentiability. For the sake of legibility, only essential mathematical details are provided.

Let $\lambda V(r)$ be a central attractive potential of finite range and strength $0<\lambda<\infty$. The potential $\lambda V(r)$ is local and is intended to represent the complex many-body problem of the interacting electron gas in the presence of a singular potential in an approximate way. $V(r)$ has the following series expansion around the origin:

$$
V(r)=\frac{v_{-1}}{r}+\sum_{j=0}^{\infty} v_{j} r^{j}, \quad v_{-1} \leqslant 0 .
$$

Some words may be needed to explain the particular notation used above. Any local analytic central potential with a Coulomb singularity at the origin can be described using the $\lambda V(r)$ form. In order to clarify the meaning of the coupling constant $\lambda$ in this context, let us take as an example the Hulthén potential $V_{\mathrm{H}}(r),{ }^{22,23}$ which will be later used for illustrative purposes:

$$
V_{\mathrm{H}}(r)=-\frac{Z}{r} \frac{\Lambda r}{e^{\Lambda r}-1},
$$

where $Z$ is the external charge and $\Lambda$ a parameter describing the screening made by the electrons. $V_{\mathrm{H}}(r)$ is expressed above in atomic units $\left(e=m_{e}=\hbar=1\right)$. The Schrödinger equation with this potential is

$$
-\frac{1}{2} \Delta \psi(\mathbf{r})-\frac{Z}{r} \frac{\Lambda r}{e^{\Lambda r}-1} \psi(\mathbf{r})=E \psi(\mathbf{r}) .
$$

Provided that $\Lambda \neq 0$, we can take $1 / \Lambda$ as a new length unit and make the following change of variables:

$$
\bar{r}=\Lambda r, \quad \lambda=Z / \Lambda, \quad \bar{E}=\Lambda^{-2} E, \quad \bar{\psi}(\overline{\mathbf{r}})=\psi(\mathbf{r}) .
$$

The Schrödinger equation can then be rewritten as

$$
-\frac{1}{2} \bar{\Delta} \bar{\psi}(\overline{\mathbf{r}})-\lambda \frac{1}{e^{\bar{r}}-1} \bar{\psi}(\overline{\mathbf{r}})=\bar{E} \bar{\psi}(\overline{\mathbf{r}}) .
$$

Hence, the form of the Hulthén potential in this new unit is the following:

$$
V_{\lambda}(\vec{r}):=\lambda V(\vec{r})=-\lambda \frac{1}{\mathrm{e}^{\bar{r}}-1} .
$$

Equations (7) and (9) illustrate the meaning of $\lambda$. The parameter $\lambda$ includes both the effect of the external charge $Z$ and the effect of the many-body electron screening through the $\Lambda$ parameter. The bare Coulomb limit $[\Lambda \rightarrow 0$ in Eq.(5)] could be recovered by just making $\lambda \rightarrow \infty$, but then the rescaling of variables is not valid anymore. The Coulomb potential, which supports an infinite number of bound states, is out of the scope of this manuscript and will be treated elsewhere, together with extended proofs and additional analysis. ${ }^{18}$ The analysis and conclusions presented here do apply to other local short-range potentials that describe the screening of charges in electronic media, such as the Thomas-Fermi potential, for instance. In practical terms, the value of $\lambda$ determines the number of bound states supported by the potential $V_{\lambda}$.

Now, let $H_{\lambda}:=-\frac{1}{2} \Delta+\lambda V$ be the Hamiltonian, and let $\psi_{\lambda, L M, E}(\mathbf{r})=R_{\lambda, L, E}(r) Y_{L}^{M}\left(\Omega_{\mathbf{r}}\right)$ denote an eigenfunction of $H_{\lambda}$ for energy $E \leqslant 0$ and unit norm: $\left\|\psi_{\lambda, L M, E}\right\|=1$. The radial wave function $u_{\lambda, L, E}(r):=r R_{\lambda, L, E}(r)$ is a regular solution of the reduced radial Schrödinger equation for $L$ waves. For $r$ $\rightarrow 0$

$$
\begin{aligned}
u_{\lambda, L, E}(r) \underset{r \rightarrow 0}{\sim} \alpha_{\lambda, L, E} r^{L+1}\left(1+\frac{\lambda v_{-1}}{L+1} r\right. \\
\left.+\frac{\lambda^{2} v_{-1}^{2}+(L+1)\left(\lambda v_{0}-E\right)}{(L+1)(2 L+3)} r^{2}+O\left(r^{3}\right)\right),
\end{aligned}
$$

where $\alpha_{\lambda, L, E}$ is a normalization constant.

From the small- $r$ behavior of the radial wave functions $u_{\lambda, L, E}(r)$ [Eq. (10)], one can show that the contribution of the bound states to the angular-averaged density $\rho_{\lambda, \mathrm{bd}}(r)$ and its radial derivatives $\rho_{\lambda, \mathrm{bd}}^{\prime}(0)$ and $\rho_{\lambda, \mathrm{bd}}^{\prime \prime}(0)$ for $r \rightarrow 0$ is

$$
\begin{aligned}
\rho_{\lambda, \mathrm{bd}}(0)= & 2 \sum_{L, E} \frac{(2 L+1)}{4 \pi}\left|R_{\lambda, L, E}(0)\right|^{2}=\frac{1}{2 \pi} \sum_{E}\left|\alpha_{\lambda, 0, E}\right|^{2}, \\
& \rho_{\lambda, \mathrm{bd}}^{\prime}(0)=2 \lambda v_{-1} \rho_{\lambda, \mathrm{bd}}(0), \\
\rho_{\lambda, \mathrm{bd}}^{\prime \prime}(0)= & \frac{1}{2 \pi}\left(\sum_{E} \frac{2}{3}\left[5 \lambda^{2} v_{-1}^{2}+2\left(\lambda v_{0}-E\right)\right]\left|\alpha_{\lambda, 0, E}\right|^{2}\right. \\
& \left.+6 \sum_{E}\left|\alpha_{\lambda, 1, E}\right|^{2}\right),
\end{aligned}
$$

where the sums run over all occupied bound states. Only those terms with $L=0$ contribute to $\rho_{\lambda, \text { bd }}(0)$ and $\rho_{\lambda, \text { bd }}^{\prime}(0)$, whereas we emphasize that both $L=0$ and $L=1$ terms need to be considered for $\rho_{\lambda, \text { bd }}^{\prime \prime}(0)$.

A similar analysis can be made for the wave functions $\psi_{\lambda, \mathbf{k}}^{+}(\mathbf{r})$ of positive energy $E=\frac{1}{2} k^{2} \geqslant 0$. Let $\psi_{\lambda, \mathbf{k}}^{+}(\mathbf{r})$ stand for an outgoing scattering state with incoming momentum $\mathbf{k}$ and normalized to the $\mathbf{k}$ scale. After expansion of $\psi_{\lambda, \mathbf{k}}^{+}(\mathbf{x})$ into partial waves, the radial function $u_{\lambda, L, k}^{+}(r)$ is the regular solution of the reduced radial Schrödinger equation, with the following behavior as $r \rightarrow 0$ : 


$$
\begin{aligned}
& u_{\lambda, L, k}^{+}(r) \underset{r \rightarrow 0}{\sim} \alpha_{\lambda, L, k}^{+} r^{L+1}\left(1+\frac{\lambda v_{-1}}{L+1} r\right. \\
&\left.+\frac{\lambda^{2} v_{-1}^{2}+(L+1)\left(\lambda v_{0}-\frac{1}{2} k^{2}\right)}{(L+1)(2 L+3)} r^{2}+O\left(r^{3}\right)\right),
\end{aligned}
$$

where

$$
\alpha_{\lambda, L, k}^{+}=\frac{1}{(2 L+1) ! !} \frac{k^{L+1}}{F_{\lambda, L}(k)} .
$$

The function $F_{\lambda, L}(k)$ is the Jost function in the $L$ channel. ${ }^{19}$

The contribution of the scattering states to the density $\rho_{\lambda, \mathrm{sc}}(r)$ can be calculated adding up all scattering states up to the Fermi level. For $r \rightarrow 0$,

$$
\begin{gathered}
\rho_{\lambda, \mathrm{sc}}(0)=2 \int_{|\mathbf{k}| \leqslant k_{\mathrm{F}}} \mathrm{d}^{3} k \frac{1}{4 \pi} \int_{S^{2}}\left|\psi_{\lambda, \mathbf{k}}^{+}(\mathbf{r})\right|^{2} \mathrm{~d} \Omega_{\mathbf{r}} \\
=\frac{1}{\pi^{2}} \int_{0}^{k_{\mathrm{F}}} \mathrm{d} k \frac{k^{2}}{\left|F_{\lambda, 0}(k)\right|^{2}}, \\
\rho_{\lambda, \mathrm{sc}}^{\prime}(0)=2 \lambda v_{-1} \rho_{\lambda, \mathrm{sc}}(0), \\
\rho_{\lambda, \mathrm{sc}}^{\prime \prime}(0)=\frac{2}{3}\left(5 \lambda^{2} v_{-1}^{2}+2 \lambda v_{0}\right) \rho_{\lambda, \mathrm{sc}}(0)+\frac{2}{3 \pi^{2}} \int_{0}^{k_{\mathrm{F}}} \mathrm{d} k k^{4} \\
\times\left[-\left|F_{\lambda, 0}(k)\right|^{-2}+\left|F_{\lambda, 1}(k)\right|^{-2}\right] .
\end{gathered}
$$

As in the bound case, only $s$-wave terms contribute to $\rho_{\lambda, \mathrm{sc}}(0)$ and $\rho_{\lambda, \mathrm{sc}}^{\prime}(0)$, whereas both $s$ and $p$ waves are relevant for $\rho_{\lambda, \mathrm{sc}}^{\prime \prime}(0)$.

Each individual (either bound or scattering) term in the overall density presents discontinuities at the thresholds. Remarkably enough they cancel each other. To prove this cancellation, we need an auxiliary lemma to express the bound state contribution in terms of the Jost functions. We know that the negative bound energies are associated with the zeroes of the Jost functions in the positive imaginary axis. Let us denote $\bar{k}=\mathrm{i} \bar{\kappa}$, such that $E=\frac{1}{2} \bar{k}^{2}=-\frac{1}{2} \kappa^{2}$ is a bound state energy. Then, $F_{\lambda, L}(\bar{k})=0$. If $\alpha_{\lambda, L, \mathrm{i} \bar{\kappa}}:=\alpha_{\lambda, L, E}$, it can be proven by an extension of a well-known result for $s$ waves $^{20,21}$ that

$$
\left|\alpha_{\lambda, L, \mathrm{i} \bar{\kappa}}\right|^{2}=(-1)^{L+1} \frac{4}{((2 L+1) ! !)^{2}} \frac{(\bar{\kappa})^{2(L+1)}}{\left[F_{\lambda, L}(-\mathrm{i} \kappa) \partial_{\kappa} F_{\lambda, L}(\mathrm{i} \kappa)\right]_{\kappa=\bar{\kappa}}} .
$$

Moreover, for real momenta $F_{\lambda, L}(k)$ vanishes only if $k=0$ and $\lambda=\lambda_{L, n}$, and thus Eqs. (14) show that arbitrarily small momenta are fully responsible for the discontinuous behavior of $\rho_{\lambda, \mathrm{sc}}(0), \rho_{\lambda, \mathrm{sc}}^{\prime}(0), \rho_{\lambda, \mathrm{sc}}^{\prime \prime}(0)$ around critical strengths.

Let us focus on the local behavior of the quantities $\left|\alpha_{\lambda, L, \mathrm{i} \kappa}\right|^{2},\left|\alpha_{\lambda, L, k}\right|^{2}$ around the thresholds $\lambda_{L, n}$ for the appearance of the $n$th bound state of angular momentum $L$. We will just pay attention to the cases relevant in our problem: $L$ $=0$ and $L=1$. From the behavior of the Jost functions for $k$ $\rightarrow 0$ and $\lambda \rightarrow \lambda_{L, n}$,

$$
\begin{gathered}
F_{\lambda, 0}(k) \sim(-1)^{n}\left[\alpha_{0, n}\left(\lambda-\lambda_{0, n}\right)+\mathrm{i} \gamma_{0, n} k+\cdots\right], \\
F_{\lambda, L>0}(k) \sim(-1)^{n}\left[\alpha_{L, n}\left(\lambda-\lambda_{L, n}\right)+\beta_{L, n} k^{2}+\mathrm{i} \gamma_{L, n} k^{3}+\cdots\right],
\end{gathered}
$$

where $\alpha_{0, n}, \gamma_{0, n}, \alpha_{L, n}, \beta_{L, n}, \gamma_{L, n}$ are strictly positive, one can prove the following propositions:

Proposition 1: The appearance of a new bound state when $\lambda$ crosses a critical value $\lambda_{L, n}$ accounts for a nonsmooth behavior of $\rho_{\lambda, \mathrm{bd}}(0), \rho_{\lambda, \mathrm{bd}}^{\prime \prime}(0)$, as functions of $\lambda$ around $\lambda_{L, n}$. Up to a $C^{1}$ function of $\lambda$,

$$
\begin{gathered}
4 \pi \rho_{\lambda, \mathrm{bd}}(0) \underset{\lambda \rightarrow \lambda_{0, n}}{\sim} 4 \alpha_{0, n} \gamma_{0, n}^{-3}\left(\lambda-\lambda_{0, n}\right) \theta\left(\lambda-\lambda_{0, n}\right), \\
4 \pi \rho_{\lambda, \mathrm{bd}}^{\prime \prime}(0) \underset{\lambda \rightarrow \lambda_{0, n}}{\sim} \quad \frac{8}{3}\left[5 \lambda_{0, n}^{2} v_{-1}^{2}+2 \lambda_{0, n} v_{0}\right] \alpha_{0, n} \gamma_{0, n}^{-3} \\
\quad \times\left(\lambda-\lambda_{0, n}\right) \theta\left(\lambda-\lambda_{0, n}\right), \\
4 \pi \rho_{\lambda, \text { bd }}^{\prime \prime}(0) \underset{\lambda \rightarrow \lambda_{1, n}}{\sim}\left[4 \alpha_{1, n} \beta_{1, n}^{-4} \gamma_{1, n}\left(\lambda-\lambda_{1, n}\right)+2 \alpha_{1, n}^{1 / 2} \beta_{1, n}^{-5 / 2} \mid \lambda\right. \\
\left.-\left.\lambda_{1, n}\right|^{1 / 2}+\frac{4}{3} \beta_{1, n}^{-1} \gamma_{1, n}^{-1}\right] \theta\left(\lambda-\lambda_{1, n}\right) .
\end{gathered}
$$

Proposition 2: In a neighborhood of $\lambda=\lambda_{L, n}$, the quantities $\rho_{\lambda, \mathrm{sc}}(0), \rho_{\lambda, \mathrm{sc}}^{\prime \prime}(0)$ behave as follows, up to a $C^{1}$ function of $\lambda$ :

$$
\begin{aligned}
& 4 \pi \rho_{\lambda, \mathrm{sc}}(0) \underset{\lambda \rightarrow \lambda_{0, n}}{\sim}-2 \alpha_{0, n} \gamma_{0, n}^{-3}\left|\left(\lambda-\lambda_{0, n}\right)\right|, \\
& 4 \pi \rho_{\lambda, \mathrm{sc}}^{\prime \prime}(0) \underset{\lambda \rightarrow \lambda_{0, n}}{\sim}-\frac{4}{3}\left(5 \lambda_{0, n}^{2} v_{-1}^{2}+2 \lambda_{0, n} v_{0}\right) \alpha_{0, n} \gamma_{0, n}^{-3} \\
& \times\left|\left(\lambda-\lambda_{0, n}\right)\right|, \\
& 4 \pi \rho_{\lambda, \mathrm{sc}}^{\prime \prime}(0) \underset{\lambda \rightarrow \lambda_{1, n}}{\sim} \frac{4}{3} \beta_{1, n}^{-1} \gamma_{1, n}^{-1} \theta\left(-\lambda+\lambda_{1, n}\right)-2 \alpha_{1, n}^{1 / 2} \beta_{1, n}^{-5 / 2} \\
& \times\left(\lambda-\lambda_{1, n}\right)^{1 / 2} \theta\left(\lambda-\lambda_{1, n}\right)-4 \alpha_{1, n} \beta_{1, n}^{-4} \gamma_{1, n} \mid \lambda \\
& -\lambda_{1, n} \mid \theta\left(-\lambda+\lambda_{1, n}\right) \text {. }
\end{aligned}
$$

Here, $\theta(x)$ is the Heavyside step function.

From these two propositions, one can easily prove the following theorem, which is the core of the present work:

Theorem: (1) The density $\rho_{\lambda}(0)$ at the origin is a $C^{1}$ function of the coupling constant $\lambda$.

(2) The cusp function $\operatorname{Cusp}(\lambda):=\rho_{\lambda}^{\prime}(0) / \rho_{\lambda}(0)$ is a linear function:

$$
\operatorname{Cusp}(\lambda)=2 v_{-1} \lambda \text {. }
$$

(3) The second radial derivative $\rho_{\lambda}^{\prime \prime}(0)$ of the density at the origin is a $C^{1}$ function of the coupling constant $\lambda$.

The third point of the theorem, i.e., the differentiability of the total curvature $\rho_{\lambda}^{\prime \prime}(0)$ as a function of $\lambda$, is actually the most relevant for our purposes. It means that no discontinuities in $\rho_{\lambda}^{\prime \prime}(0)$ are expected whenever $\lambda$ reaches the threshold for the appearance of a new bound state.

In the following, we illustrate the smoothness of the elec- 

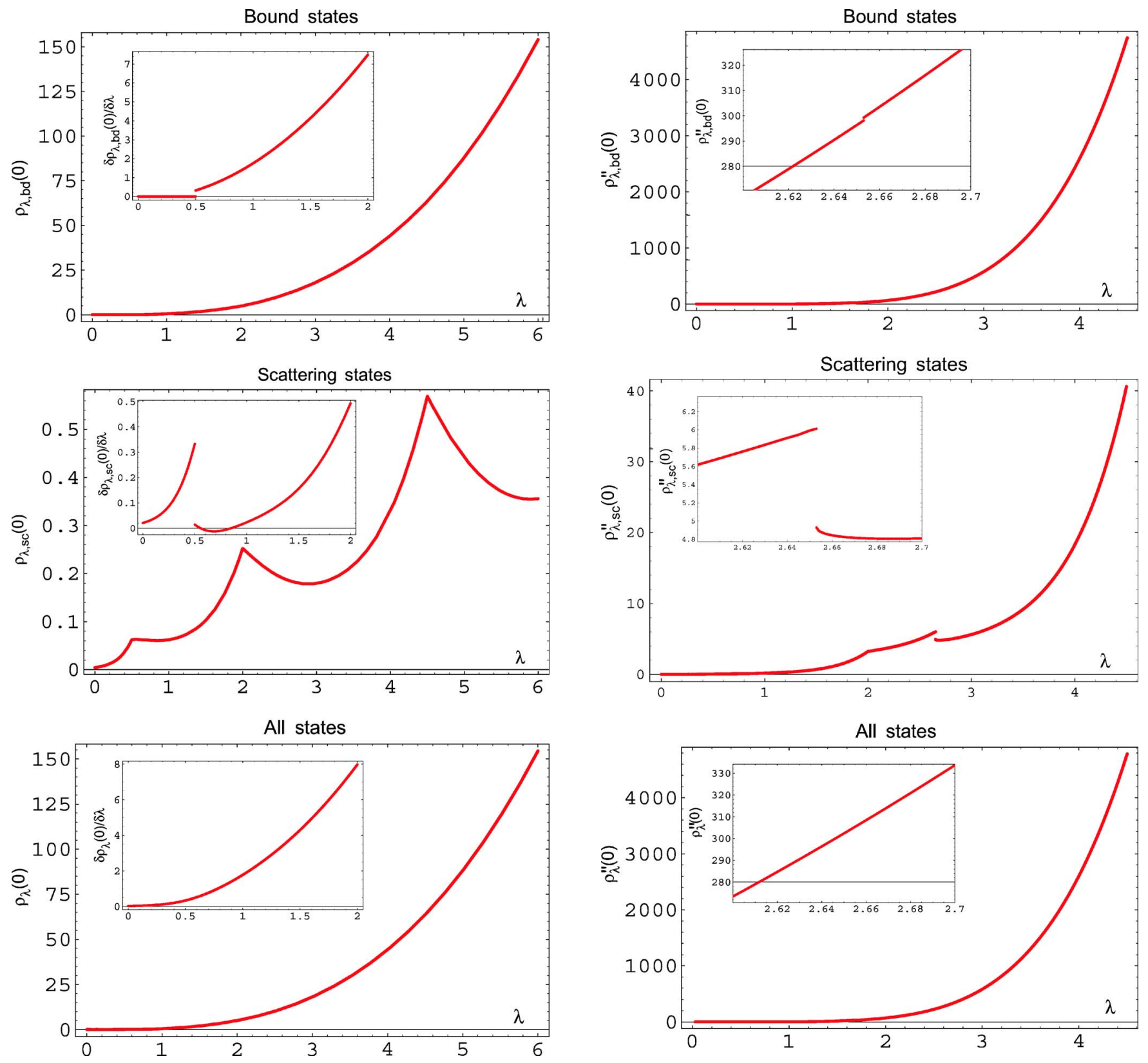

FIG. 1. (Color online) Partial components of the electronic density at the origin $\rho_{\lambda, \mathrm{bd}}(0)$ (upper panel) and $\rho_{\lambda, \mathrm{sc}}(0)$ (middle panel) as a function of the coupling constant $\lambda$ for a Hulthén potential $\lambda V(r)=-\lambda /\left(\mathrm{e}^{r}-1\right)$. The total density $\rho_{\lambda}(0):=\rho_{\lambda, \mathrm{bd}}(0)+\rho_{\lambda, \mathrm{sc}}(0)$ is shown as well in the lower panel. The insets show the derivatives of these functions with respect to $\lambda\left[\delta \rho_{\lambda, \text { bd }}(0) / \delta \lambda, \delta \rho_{\lambda, \mathrm{sc}}(0) / \delta \lambda\right.$, and $\delta \rho_{\lambda}(0) / \delta \lambda$ in the upper, middle, and lower panels, respectively] in the vicinity of the threshold $\lambda=\lambda_{0,1}$ for the appearance of the first $(L=0)$-bound state.

tronic density at the origin, as well as the smoothness of the curvature by making use of the Hulthén potential [Eq. (9)]. For this potential, explicit analytical expressions are available for the wave functions with $L=0 .{ }^{24}$ The corresponding eigenenergies of the bound states with $L=0$ are $E_{n 0}=-(\lambda / n$ $-n / 2)^{2} / 2$, with $1 \leqslant n<\sqrt{2 \lambda}$. Therefore there are threshold values $\lambda_{0, n}:=\frac{1}{2} n^{2}, n \geqslant 1$, marking the appearance of a new $L=0$ eigenstate for any $\lambda>\lambda_{0, n}$. The normalization constants

FIG. 2. (Color online) Partial components of the curvature at the origin $\rho_{\lambda, \text { bd }}^{\prime \prime}(0)$ (upper panel) and $\rho_{\lambda, \text { sc }}^{\prime \prime}(0)$ (middle panel) as a function of the coupling constant $\lambda$ for a Hulthén potential $\lambda V(r)=$ $-\lambda /\left(\mathrm{e}^{r}-1\right)$. The total curvature $\rho_{\lambda}^{\prime \prime}(0):=\rho_{\lambda, \mathrm{bd}}^{\prime \prime}(0)+\rho_{\lambda, \mathrm{sc}}^{\prime \prime}(0)$ is shown as well in the lower panel. The insets show the same functions in the vicinity of the threshold $\lambda=\lambda_{1,1}$ for the appearance of the first $(L=1)$-bound state.

$\left|\alpha_{\lambda, 0, E_{n 0}}\right|^{2}$ and the Jost function $F_{\lambda, 0}$ are also analytical. For bound eigenfunctions with $L \geqslant 1$, one has to resort to numerical computation. For instance, $\lambda_{1, n}=\{2.6530,5.3626$, $9.0504, \ldots\}$ for $n=1,2,3 \ldots$ The constants $\left|\alpha_{\lambda, 1, E_{n 1}}\right|^{2}$ and the function $F_{\lambda, 1}(k)$ also require numerical computation.

Without loss of generality, we fix the Fermi level to $k_{F}$ $=\frac{1}{2}$ for illustrative purposes. The smoothness does not depend on the particular value of $k_{F}$. In Fig. 1 we show the behavior of the partial components of the density $\rho_{\lambda, \mathrm{bd}}(0)$ and $\rho_{\lambda, \mathrm{sc}}(0)$, as well as the total density $\rho_{\lambda}(0):=\rho_{\lambda, \mathrm{bd}}(0)$ 
$+\rho_{\lambda, \mathrm{sc}}(0)$ as functions of $\lambda$. Figure 1 shows clearly that the bound state contribution $\rho_{\lambda, \text { bd }}(0)$ and the scattering state contribution $\rho_{\lambda, \mathrm{sc}}(0)$ present cusp points as functions of $\lambda$ at the values in which a new bound state appears. The cusps are washed out when added up to obtain the all state contribution $\rho_{\lambda}(0)$. The lack of differentiability of $\rho_{\lambda, \text { bd }}(0)$ hardly leaps to the eye due to the dominance of the bound states contribution. To render it visible we show in the insets the behavior of the derivatives (with respect to $\lambda$ ) $\delta \rho_{\lambda, \text { bd }}(0) / \delta \lambda$, $\delta \rho_{\lambda, \mathrm{sc}}(0) / \delta \lambda$, and $\delta \rho_{\lambda}(0) / \delta \lambda$, in a neighborhood of the first $L=0$ threshold $\lambda_{0,1}$.

The functions $\rho_{\lambda, \text { bd }}^{\prime \prime}(0), \quad \rho_{\lambda, \text { sc }}^{\prime \prime}(0)$, and $\rho_{\lambda}^{\prime \prime}(0):=\rho_{\lambda, \text { bd }}^{\prime \prime}(0)$ $+\rho_{\lambda, \mathrm{sc}}^{\prime \prime}(0)$ are shown in Fig. 2 . The discontinuities of $\rho_{\lambda, \mathrm{bd}}^{\prime \prime}(0)$ are again not apparent in this figure due to the scale used. A particular case (the threshold for the appearance of the first bound state with $L=1$ ) is shown in the inset. Similar insets are used in the plots of $\rho_{\lambda, \mathrm{sc}}^{\prime \prime}(0)$ and $\rho_{\lambda}^{\prime \prime}(0)$. In them, the lack of differentiability of $\rho_{\lambda, \mathrm{bd}}^{\prime \prime}(0)$ and $\rho_{\lambda, \mathrm{sc}}^{\prime \prime}(0)$ is clearly visible. Figure 2 shows the perfect cancellation of the discontinuities in the two partial components $\rho_{\lambda, \text { bd }}^{\prime \prime}(0)$ and $\rho_{\lambda, s c}^{\prime \prime}(0)$, which renders the function $\rho_{\lambda}^{\prime \prime}(0)$ a differentiable function of $\lambda$ even at the values of $\lambda$ for which new bound states arise.

Summarizing, we have shown in a transparent, though rigorous, manner that $\rho_{\lambda}(r), \rho_{\lambda}^{\prime}(r)$, and $\rho_{\lambda}^{\prime \prime}(r)$ are differentiable functions of $\lambda$ at $r=0$, for any $\lambda \geqslant 0$, where $\lambda$ is a coupling constant in an attractive, screened potential $\lambda V(r)$, such as a Coulomb-like potential embedded in a polarizable medium. Particularly, the smooth behavior of $\rho_{\lambda}^{\prime \prime}(r)$ at $r=0$ shows that the curvature does not provide a criterion for the bound-to-free transition in ground-state screening in para- magnetic metallic environments. The case of a spin-polarized electron gas would require some minor modifications in our theoretical development, but should lead to the same conclusions with respect to the smoothness of the curvature for the spin-polarized partial electron densities.

The smoothness of $\rho_{\lambda}(r)$ and its derivatives at the Coulomb cusp is relevant to any many-body electronic structure calculation based on trial wave functions. More specifically, it provides a strong test for those trying to describe transitions from localized to continuum states in a metallic environment. For instance, an exciting new field for which our results could be helpful is the study of metallic hydrogen at pressures beyond molecular dissociation..$^{25,26}$ In this kind of problems, the value of the curvature at the origin is strongly related to sum rules for the partial structure factors. ${ }^{16} \mathrm{~A}$ temperature-dependent extension of the analysis, via modifications in the momentum distribution function, may also have applications in plasma physics.

\section{ACKNOWLEDGMENTS}

The authors are thankful to Neil W. Ashcroft for his encouraging comments on this work. Financial support by the Basque Departamento de Educación, Universidades e Investigación, the University of the Basque Country UPV/EHU (Grant No. 9/UPV 00206.215-13639/2001), the Spanish MEC (Grant Nos. FIS2004-06490-C03-00 and FPA200402602), and the EU Network of Excellence NANOQUANTA (Grant No. NMP4-CT-2004-500198) is acknowledged. The work of I.N. was partially supported by the Hungarian OTKA (Grant Nos. T046868 and T049571).
${ }^{1}$ P. Hohenberg and W. Kohn, Phys. Rev. 136, B864 (1964).

${ }^{2}$ W. Kohn and L. J. Sham, Phys. Rev. 140, A1133 (1965).

${ }^{3}$ W. Kohn, Rev. Mod. Phys. 71, 1253 (1998).

${ }^{4}$ E. Zaremba, L. M. Sander, H. B. Shore, and J. H. Rose, J. Phys. F: Met. Phys. 7, 1763 (1977).

${ }^{5}$ M. J. Puska and R. M. Nieminen, Phys. Rev. B 27, 6121 (1983).

${ }^{6}$ P. M. Echenique, F. Flores, and R. H. Ritchie, in Solid State Physics Series, edited by H. Ehrenreich and D. Turnbull (Academic Press, New York, 1990), Vol. 43, p. 229.

${ }^{7}$ R. Díez Muiño, M. Alducin, and P. M. Echenique, Phys. Rev. B 67, 121101(R) (2003).

${ }^{8}$ M. Alducin, A. Arnau, and I. Nagy, Phys. Rev. A 68, 014701 (2003).

${ }^{9}$ T. Kato, Commun. Pure Appl. Math. 10, 151 (1957).

${ }^{10}$ E. J. Steiner, J. Chem. Phys. 39, 2365 (1963).

${ }^{11}$ J. C. Kimball, Phys. Rev. A 7, 1648 (1973).

${ }^{12}$ R. Peierls, Surprises in Theoretical Physics (Princeton University Press, Princeton, 1979), pp. 137-146.

${ }^{13}$ I. Nagy and B. Apagyi, Adv. Quantum Chem. 46, 268 (2004).

${ }^{14}$ W. Kohn and C. Majumdar, Phys. Rev. 138, A1617 (1965).

${ }^{15}$ R. O. Esquivel, J. Chen, M. J. Stott, R. P. Sagar, and V. H. Smith,
Phys. Rev. A 47, 936 (1993).

${ }^{16}$ K. Moulopoulos and N. W. Ashcroft, Phys. Rev. B 56, 4439 (1997).

${ }^{17}$ S. Doniach and F. H. Sondheimer, Green's Functions for Solid State Physicists (Benjamin, London, 1974), pp. 184-189.

${ }^{18}$ A. Galindo, I. Nagy, R. Díez Muiño, and P. M. Echenique (unpublished).

${ }^{19}$ A. Galindo and P. Pascual, Quantum Mechanics (Springer-Verlag, Berlin, 1991), Vol. II.

${ }^{20}$ R. J. Taylor, Scattering Theory (Wiley, New York, 1972).

${ }^{21}$ R. G. Newton, Scattering Theory of Waves and Particles (Springer-Verlag, New York, 1982).

${ }^{22}$ L. Hulthén, Ark. Mat., Astron. Fys. 28, 1 (1942).

${ }^{23}$ J. Lindhard and A. Winter, Nucl. Phys. A 166, 413 (1971).

${ }^{24}$ R. Dutt, K. Chowdhury, and Y. P. Varshni, J. Phys. A 18, 1379 (1985).

${ }^{25}$ C. Pierleoni, D. M. Ceperley, and M. Holzmann, Phys. Rev. Lett. 93, 146402 (2004).

${ }^{26}$ E. Babaev, A. Sudb $\varnothing$, and N. W. Ashcroft, Nature (London) 431, 666 (2004). 